\title{
Global Small Solutions to a Complex Fluid Model in 3D
}

\author{
Fanghua Lin ${ }^{1}$, Ting Zhang ${ }^{2}$ \\ 1. Courant Institute, New York University, New York, NY 10012, USA \\ 2. Department of Mathematics, Zhejiang University, Hangzhou 310027, China
}

\begin{abstract}
In this paper, we provide a much simplified proof of the main result in [14] concerning the global existence and uniqueness of smooth solutions to the Cauchy problem for a 3D incompressible complex fluid model under the assumption that the initial data are close to some equilibrium states. Beside the classical energy method, the interpolating inequalities and the algebraic structure of the equations coming from the incompressibility of the fluid are crucial in our arguments. We combine the energy estimates with the $L^{\infty}$ estimates for time slices to deduce the key $L^{1}$ in time estimates. The latter is responsible for the global in time existence.
\end{abstract}

\section{Introduction.}

In this paper, we consider the global existence of classical solutions to the following simple model for a complex fluid flows

$$
\left\{\begin{array}{l}
\partial_{t} \phi+v \cdot \nabla \phi=0, \quad(t, x) \in \mathbb{R}^{+} \times \mathbb{R}^{3}, \\
\partial_{t} v+v \cdot \nabla v-\Delta v+\nabla p=-\operatorname{div}[\nabla \phi \otimes \nabla \phi], \\
\operatorname{div} v=0, \\
\left.(\phi, v)\right|_{t=0}=\left(\phi_{0}, v_{0}\right) .
\end{array}\right.
$$

Here $\phi, v=\left(v_{1}, v_{2}, v_{3}\right)^{\top}$ and $p$ denote the scalar potential, velocity field and scalar pressure of the fluid respectively. As in [14, we shall consider the initial data $\left(\phi_{0}, v_{0}\right)$ is close to a non-trivial equilibrium, e.g. $\left(\phi_{0}, v_{0}\right) \simeq\left(x_{3},(0,0,0)^{\top}\right)$. Note that instead of $x_{3}$, any non-constant linear functions would work as well by our method (cf. 14 for explanations). What would be important is that $\nabla \phi_{0}$ is close to a constant non-zero vector field in a suitable way. Thus, we may write $(\phi, v)=\left(x_{3}+\psi, v\right)$, and substitute it into (1.1) with $x \in \mathbb{R}^{3}$, to obtain the following equivalent system for $(\psi, v)$,

$$
\left\{\begin{array}{l}
\partial_{t} \psi+v \cdot \nabla \psi+v_{3}=0, \quad(t, x) \in \mathbb{R}^{+} \times \mathbb{R}^{3}, \\
\partial_{t} v_{h}+v \cdot \nabla v_{h}-\Delta v_{h}+\nabla_{h} p+\nabla_{h} \partial_{3} \psi=-\operatorname{div}\left[\nabla_{h} \psi \otimes \nabla \psi\right], \\
\partial_{t} v_{3}+v \cdot \nabla v_{3}-\Delta v_{3}+\partial_{3} p+\left(\Delta+\partial_{3}^{2}\right) \psi=-\operatorname{div}\left[\partial_{3} \psi \nabla \psi\right], \\
\operatorname{div} v=0, \\
\left.(\psi, v)\right|_{t=0}=\left(\psi_{0}, v_{0}\right),
\end{array}\right.
$$

where $v_{h}=\left(v_{1}, v_{2}\right)^{\top}, \nabla_{h}=\left(\partial_{1}, \partial_{2}\right)^{\top}$. For the rest of the paper we shall work on the equations (1.2).

We remark that the nonlinear hyperbolic-parabolic system (1.1) has been used for describing many fluid dynamic models, see 3, 4, 12. Indeed, when $\phi=\left(\phi_{1}, \phi_{2}\right)^{\top}$ is a vector-valued function on $\mathbb{R}^{+} \times \mathbb{R}^{2}$ with $\operatorname{det}(\nabla \phi)=1$, the system (1.1) is equivalent to the well-known Oldroyd-B model for viscoelastic fluids equations, see [8, 11, 13, 17. It is also closely related to the evolution equation of nematic liquid crystal as well as the diffusive sharp interface motion and immersed boundary in flow fields [10, see also the recent survey article [12. In [14], authors used the system (1.1) as a toy model for the 3D incompressible viscous and non-resistive MHD system.

In fact, the system (1.1) is exactly the incompressible MHD equations with zero magnetic diffusion when the space dimension is two. Recall that the 2D incompressible MHD system reads,

$$
\left\{\begin{array}{l}
\partial_{t} b+v \cdot \nabla b-\eta \Delta b=b \cdot \nabla v, \quad(t, x) \in \mathbb{R}^{+} \times \mathbb{R}^{2}, \\
\partial_{t} v+v \cdot \nabla v-\nu \Delta v+\nabla p=b \cdot \nabla b, \\
\operatorname{div} v=\operatorname{div} b=0, \\
\left.b\right|_{t=0}=b_{0},\left.v\right|_{t=0}=v_{0},
\end{array}\right.
$$


where $b=\left(b_{1}, b_{2}\right)^{\top}, v=\left(v_{1}, v_{2}\right)^{\top}$ and $p$ denote the magnetic field, velocity field and scalar pressure of the fluid respectively. In (1.3), the condition $\operatorname{div} b=0$ implies the existence of a scalar function $\phi$ such that $b=\left(\partial_{2} \phi,-\partial_{1} \phi\right)^{\top}$, and the corresponding system becomes the following 2D incompressible MHD type system,

$$
\left\{\begin{array}{l}
\partial_{t} \phi+v \cdot \nabla \phi-\eta \Delta \phi=0, \quad(t, x) \in \mathbb{R}^{+} \times \mathbb{R}^{2}, \\
\partial_{t} v+v \cdot \nabla v-\Delta v+\nabla p=-\operatorname{div}[\nabla \phi \otimes \nabla \phi], \\
\operatorname{div} v=0, \\
\left.(\phi, v)\right|_{t=0}=\left(\phi_{0}, v_{0}\right) .
\end{array}\right.
$$

There are some global wellposedness results for the system (1.3), see [6, 18, for the case when $\eta>0$ and $\nu>0,2$ for the case when $\eta>0$ and $\nu=0$, as well as the case with mixed partial dissipation and additional (artificial) magnetic diffusion. In [1, authored considered the case when $\eta=\nu=0$ and the initial data $\left(b_{0}, v_{0}\right)$ close to the equilibrium state $\left(B_{0}, 0\right)$. In $[13$, the case when $\eta=0, \nu>0$ was studied. Under the assumption that the initial data $\left(b_{0}, v_{0}\right)$ is close to the equilibrium state $\left((1,0)^{\top}, 0\right)$, the global wellposedness was proven. We should note that authors observed in 1 that the fluctuations $v+b-B_{0}$ and $v-b+B_{0}$ propagate along the $B_{0}$ magnetic field in opposite directions. Thus, a strong enough magnetic field will reduce the nonlinear interactions and prevent formations of strong gradients [1, 7, 9]. Unfortunately, the method applied in [1] is purely hyperbolic (characteristic method) and hence could not be applied in our case.

In [14, using the anisotropic Littlewood-Paley analysis, the first author and Ping Zhang [14] proved a global wellposedness result of the system (1.1). The arguments involved, despite its general interests, were rather complicated. The aim of this note is to give a new and simple proof, which involves only the energy estimate method, interpolating inequalities and couple elementary observations.

Theorem 1.1. Assume that the initial data $\left(\psi_{0}, v_{0}\right)$ satisfy $\left(\nabla \psi_{0}, v_{0}\right) \in H^{2}\left(\mathbb{R}^{3}\right) \times H^{2}\left(\mathbb{R}^{3}\right)$, $\operatorname{div} v_{0}=0$, then there exists a positive constant $c_{0}$ such that if

$$
B_{0}=\left\|\nabla \psi_{0}\right\|_{H^{2}}+\left\|v_{0}\right\|_{H^{2}} \leq c_{0},
$$

then the system (1.2) has a unique global solution $(\psi, v, \nabla p) \in F^{2}$ satisfying

$$
B_{T}^{2}=\|v\|_{L^{\infty}\left([0, T] ; H^{2}\right)}^{2}+\|\nabla \psi\|_{L^{\infty}\left([0, T] ; H^{2}\right)}^{2}+\|\nabla v\|_{L^{2}\left([0, T] ; H^{2}\right)}^{2}+\left\|\nabla_{h} \nabla \psi\right\|_{L^{2}\left([0, T] ; H^{1}\right)}^{2} \leq C B_{0}^{2},
$$

and

$$
\|\nabla p\|_{L^{\infty}\left([0, T] ; H^{1}\right)} \leq C B_{0},
$$

for all $T>0$, where $C$ is a positive constant independent of $T$,

$$
F^{n}=\left\{\begin{array}{l|l}
(\psi, v, \nabla p) \mid \begin{array}{l}
(\nabla \psi, v, \nabla p) \in C\left([0, \infty) ; H^{n} \times H^{n}\right) \times C\left([0, \infty) ; H^{n-1}\right), \\
(\nabla h \nabla \psi, \nabla v) \in L^{2}\left([0, \infty) ; H^{n-1} \times H^{n}\right) .
\end{array}
\end{array}\right\}
$$

We note that under the assumptions of Theorem 1.1, if $\left(\nabla \psi_{0}, v_{0}\right) \in H^{n}\left(\mathbb{R}^{3}\right) \times H^{n}\left(\mathbb{R}^{3}\right), n \geq 3$, then we can easily obtain that $(\psi, v, \nabla p) \in F^{n}$ and omit the details.

There are three key technical points in our proofs:

(1) interpolating estimates, see for example, Lemma 2.1

(2) using the algebraic structure: $\operatorname{div} v=0$ to inter-changing the estimates for the vertical $\left(\partial_{3}\right)$ and the horizontal $\left(\nabla_{h}\right)$ derivatives;

(3) using the first equation of (1.2) to reduce " $L^{1}$ in time estimates" (14]) which is the key to the global existence result to "energy estimates and $L^{\infty}$ estimates for time slices" that are relatively easy to obtain.

In fact, the basic strategy for the proofs is rather clear. Using the basic energy laws, one reduces the problems to estimating certain terms of particular forms. For example, one of the difficulties of the proofs would be to control the following type term,

$$
\int_{0}^{T} \int_{\mathbb{R}^{3}} \partial_{3} v_{3}\left(\partial_{3}^{3} \psi\right)^{2} d x d t
$$


Since the horizontal derivatives of $\psi, \nabla_{h} \psi$ decay faster than $\partial_{3} \psi$ (by energy laws), in [14 authors explored such anisotropic behavior by using the anisotropic Littlewood-Paley theory to conclude the key estimate that $v_{3} \in L^{1}\left(\mathbb{R}^{+} ; \operatorname{Lip}\left(\mathbb{R}^{3}\right)\right)$. Here we will show that first by interpolating inequalities $\nabla \psi \in L_{T}^{4}\left(L^{\infty}\right)$ in Lemma 2.1. Then we use the first equation of $(1.2)_{1}$ twice and proceed the estimates as follows:

$$
\begin{aligned}
& \left|\int_{0}^{T} \int_{\mathbb{R}^{3}} \partial_{3} v_{3}\left(\partial_{3}^{3} \psi\right)^{2} d x d t\right| \\
= & \left|\int_{0}^{T} \int_{\mathbb{R}^{3}} \partial_{3}\left(\partial_{t} \psi+v \cdot \nabla \psi\right)\left(\partial_{3}^{3} \psi\right)^{2} d x d t\right| \\
\leq & \left|\int_{\mathbb{R}^{3}} \partial_{3} \psi\left(\partial_{3}^{3} \psi\right)^{2} d x\right|_{0}^{T}|+| \int_{0}^{T} \int_{\mathbb{R}^{3}} \partial_{3} v_{3} \partial_{3} \psi\left(\partial_{3}^{3} \psi\right)^{2} d x d t \mid+\ldots \\
= & \ldots+\left|\int_{0}^{T} \int_{\mathbb{R}^{3}} \partial_{3}\left(\partial_{t} \psi+v \cdot \nabla \psi\right) \partial_{3} \psi\left(\partial_{3}^{3} \psi\right)^{2} d x d t\right|+\ldots \\
\leq & \ldots+C\|\nabla \psi\|_{L_{T}^{4}\left(L^{\infty}\left(\mathbb{R}^{3}\right)\right)}^{2} \mid\|\nabla v\|_{L_{T}^{2}\left(L^{2}\left(\mathbb{R}^{3}\right)\right)}\left\|\partial_{3}^{3} \psi\right\|_{L_{T}^{\infty}\left(L^{2}\left(\mathbb{R}^{3}\right)\right)}^{2}+\ldots
\end{aligned}
$$

We refere the details to Lemma 2.5. This is a simple idea works well for the issue concerning various anisotropic dissipative system similar to (1.2).

We also note that in a recent preprint [13] authores embedded the system (1.3) with $\eta=0$ into a 2D viscoelastic fluid system. Then they use equations in Lagrangian coordinates and the anisotropic Littlewood-Paley analysis techniques, to obtain a global wellposedness result. One can apply the methods in this paper to obtain similar results as theirs.

The organization of this paper can be as the following: we shall present some a priori estimates in Section 2 and prove Theorem 1.1 in Section 3 .

Let us complete this section by the notation we shall use in this paper.

Notation. We shall denote by $(a \mid b)$ the $L^{2}$ inner product of $a$ and $b$, and $(a \mid b)_{H^{s}}$ the standard $H^{s}$ inner product of $a$ and $b$. Finally, we denote $L_{T}^{p}\left(L_{h}^{q}\left(L_{v}^{r}\right)\right)$ the space $L^{p}\left([0, T] ; L^{q}\left(\mathbb{R}_{x_{1}} \times \mathbb{R}_{x_{2}} ; L^{r}\left(\mathbb{R}_{x_{3}}\right)\right)\right)$, $C_{T}(X)$ the space $C([0, T] ; X)$.

\section{$2 \quad$ A priori estimates}

In this section, we prove a set of a priori estimates which are crucial for the global existence of solutions for the system (1.2). We begin with the following Gagliardo-Nirenberg-Sobolev type estimate, see [16].

Lemma 2.1. If the function $\psi$ satisfies that $\nabla \psi \in L_{T}^{\infty}\left(H^{2}\left(\mathbb{R}^{3}\right)\right)$ and $\nabla_{h} \nabla \psi \in L_{T}^{2}\left(H^{1}\left(\mathbb{R}^{3}\right)\right)$, then there hold

$$
\begin{gathered}
\|\nabla \psi\|_{L_{T}^{4}\left(L^{4}\left(\mathbb{R}^{3}\right)\right)} \leq C\left\|\nabla_{h} \nabla \psi\right\|_{L_{T}^{2}\left(L^{2}\left(\mathbb{R}^{3}\right)\right)}^{\frac{1}{2}}\|\nabla \psi\|_{L_{T}^{\infty}\left(H^{1}\left(\mathbb{R}^{3}\right)\right)}^{\frac{1}{2}}, \\
\left\|\nabla^{2} \psi\right\|_{L_{T}^{4}\left(L^{4}\left(\mathbb{R}^{3}\right)\right)} \leq C\left\|\nabla_{h} \nabla \psi\right\|_{L_{T}^{2}\left(H^{1}\left(\mathbb{R}^{3}\right)\right)}^{\frac{1}{2}}\|\nabla \psi\|_{L_{T}^{\infty}\left(H^{2}\left(\mathbb{R}^{3}\right)\right)}^{\frac{1}{2}}, \\
\|\nabla \psi\|_{L_{T}^{4}\left(L^{\infty}\left(\mathbb{R}^{3}\right)\right)} \leq C\left\|\nabla_{h} \nabla \psi\right\|_{L_{T}^{2}\left(H^{1}\left(\mathbb{R}^{3}\right)\right)}^{\frac{1}{2}}\|\nabla \psi\|_{L_{T}^{\infty}\left(H^{2}\left(\mathbb{R}^{3}\right)\right)}^{\frac{1}{2}},
\end{gathered}
$$

where $C$ is a positive constant independent of $T$.

Proof. Using Gagliardo-Nirenberg-Sobolev's inequality and Minkowski's inequality, we obtain

$$
\begin{aligned}
\|\nabla \psi\|_{L_{T}^{4}\left(L^{4}\left(\mathbb{R}^{3}\right)\right)} & \leq C\|\| \nabla \psi\left\|_{L_{h}^{2}}^{\frac{1}{2}}\right\| \nabla \nabla_{h} \psi\left\|_{L_{h}^{2}}^{\frac{1}{2}}\right\|_{L_{T}^{4}\left(L_{v}^{4}\right)} \\
& \leq C\|\nabla \psi\|_{L_{T}^{\infty}\left(L_{v}^{\infty}\left(L_{h}^{2}\right)\right)}^{\frac{1}{2}}\left\|\nabla \nabla_{h} \psi\right\|_{L^{2}\left(L^{2}\left(\mathbb{R}^{3}\right)\right)}^{\frac{1}{2}}\left\|\nabla \nabla_{h} \psi\right\|_{L^{2}\left(L^{2}\left(\mathbb{R}^{3}\right)\right)}^{\frac{1}{2}} \\
& \leq C\|\nabla \psi\|_{L_{T}^{\infty}\left(L_{h}^{2}\left(L_{v}^{\infty}\right)\right)}^{\frac{1}{2}}
\end{aligned}
$$




$$
\begin{aligned}
& \leq C\|\| \nabla \psi\left\|_{L_{v}^{2}}^{\frac{1}{2}}\right\| \nabla \partial_{3} \psi\left\|_{L_{v}^{2}}^{\frac{1}{2}}\right\|_{L_{T}^{\infty}\left(L_{h}^{2}\right)}^{\frac{1}{2}}\left\|\nabla \nabla_{h} \psi\right\|_{L^{2}\left(L^{2}\left(\mathbb{R}^{3}\right)\right)}^{\frac{1}{2}} \\
& \leq C\|\nabla \psi\|_{L_{T}^{\infty}\left(L^{2}\left(\mathbb{R}^{3}\right)\right)}^{\frac{1}{4}}\left\|\nabla \partial_{3} \psi\right\|_{L_{T}^{\infty}\left(L^{2}\left(\mathbb{R}^{3}\right)\right)}^{\frac{1}{4}}\left\|\nabla \nabla_{h} \psi\right\|_{L_{T}^{2}\left(L^{2}\left(\mathbb{R}^{3}\right)\right)}^{\frac{1}{2}} \\
& \leq C\left\|\nabla_{h} \nabla \psi\right\|_{L_{T}^{2}\left(L^{2}\left(\mathbb{R}^{3}\right)\right)}^{\frac{1}{2}}\|\nabla \psi\|_{L_{T}^{\infty}\left(H^{1}\left(\mathbb{R}^{3}\right)\right)}^{\frac{1}{2}} .
\end{aligned}
$$

This proves (2.1). Inequality (2.2) is a direct consequence of (2.1). Combining (2.1)-(2.2) with GagliardoNirenberg-Sobolev's inequality again, we obtain (2.3).

By taking divergence of the $v$ equation of (1.2), we can express the pressure function $p$ via

$$
p=-2 \partial_{3} \psi+\sum_{i, j=1}^{3}(-\Delta)^{-1}\left[\partial_{i} v_{j} \partial_{j} v_{i}+\partial_{i} \partial_{j}\left(\partial_{i} \psi \partial_{j} \psi\right)\right] .
$$

As in [14, we substitute (2.5) into (1.2) to obtain

$$
\left\{\begin{array}{l}
\partial_{t} \psi+v \cdot \nabla \psi+v_{3}=0, \quad(t, x) \in \mathbb{R}^{+} \times \mathbb{R}^{3}, \\
\partial_{t} v_{h}+v \cdot \nabla v_{h}-\Delta v_{h}-\nabla_{h} \partial_{3} \psi=f^{h} \\
=-\sum_{i, j=1}^{3} \nabla_{h}(-\Delta)^{-1}\left[\partial_{i} v_{j} \partial_{j} v_{i}+\partial_{i} \partial_{j}\left(\partial_{i} \psi \partial_{j} \psi\right)\right]-\sum_{j=1}^{3} \partial_{j}\left[\nabla_{h} \psi \partial_{j} \psi\right], \\
\partial_{t} v_{3}+v \cdot \nabla v_{3}-\Delta v_{3}+\Delta_{h} \psi=f^{v} \\
\quad=-\sum_{i, j=1}^{3} \partial_{3}(-\Delta)^{-1}\left[\partial_{i} v_{j} \partial_{j} v_{i}+\partial_{i} \partial_{j}\left(\partial_{i} \psi \partial_{j} \psi\right)\right]-\sum_{j=1}^{3} \partial_{j}\left[\partial_{3} \psi \partial_{j} \psi\right], \\
\operatorname{div} v=0, \\
\left.(\psi, v)\right|_{t=0}=\left(\psi_{0}, v_{0}\right) .
\end{array}\right.
$$

Here, $\Delta_{h}=\partial_{x_{1}}^{2}+\partial_{x_{2}}^{2}$.

The next Lemma is a standard energy estimate.

Lemma 2.2. Let $(\psi, v)$ be sufficiently smooth functions which solve (1.2), then there holds

$$
\begin{aligned}
& \frac{d}{d t}\left\{\frac{1}{2}\left(\|v\|_{H^{2}}^{2}+\|\nabla \psi\|_{H^{2}}^{2}+\frac{1}{4}\|\Delta \psi\|_{H^{1}}^{2}\right)+\frac{1}{4}\left(v_{3} \mid \Delta \psi\right)_{H^{1}}\right\} \\
& +\|\nabla v\|_{H^{2}}^{2}-\frac{1}{4}\left\|\nabla v_{3}\right\|_{H^{1}}^{2}+\frac{1}{4}\left\|\nabla \nabla_{h} \psi\right\|_{H^{1}}^{2} \\
= & -(v \cdot \nabla v \mid v)_{H^{2}}+(v \cdot \nabla \psi \mid \Delta \psi)_{H^{2}}-(\operatorname{div}(\nabla \psi \otimes \nabla \psi) \mid v)_{H^{2}}-\frac{1}{4}\left(v \cdot \nabla v_{3} \mid \Delta \psi\right)_{H^{1}} \\
& +\frac{1}{4}\left(f^{v} \mid \Delta \psi\right)_{H^{1}}+\frac{1}{4}\left(\nabla v_{3} \mid \nabla(v \cdot \nabla \psi)\right)_{H^{1}}-\frac{1}{4}(\Delta(v \cdot \nabla \psi) \mid \Delta \psi)_{H^{1}} .
\end{aligned}
$$

Proof. Taking the standard $H^{2}$ inner product of $(1.2)_{2,3}$ with $v$ and then using the integration by parts, we have

$$
\begin{aligned}
& \frac{1}{2} \frac{d}{d t}\|v\|_{H^{2}}^{2}+(v \cdot \nabla v \mid v)_{H^{2}}+\|\nabla v\|_{H^{2}}^{2} \\
= & -\left(\nabla_{h} \partial_{3} \psi \mid v_{h}\right)_{H^{2}}-\left(\left(\Delta+\partial_{3}^{2}\right) \psi \mid v_{3}\right)_{H^{2}}-(\operatorname{div}(\nabla \psi \otimes \nabla \psi) \mid v)_{H^{2}} .
\end{aligned}
$$

Since $\operatorname{div} v=0$, the integration by parts gives

$$
\begin{aligned}
& -\left(\nabla_{h} \partial_{3} \psi \mid v_{h}\right)_{H^{2}}=\left(\partial_{3} \psi \mid \operatorname{div}_{h} v_{h}\right)_{H^{2}} \\
= & -\left(\partial_{3} \psi \mid \partial_{3} v_{3}\right)_{H^{2}}=\left(\partial_{3}^{2} \psi \mid v_{3}\right)_{H^{2}} .
\end{aligned}
$$

From $(1.2)_{1}$, we have

$$
-\left(\Delta \psi \mid v_{3}\right)_{H^{2}}=\left(\Delta \psi \mid\left(\partial_{t} \psi+v \cdot \nabla \psi\right)\right)_{H^{2}}
$$




$$
=-\frac{1}{2} \frac{d}{d t}\|\nabla \psi\|_{H^{2}}^{2}+(\Delta \psi \mid v \cdot \nabla \psi)_{H^{2}} .
$$

Combining (2.8)-(2.10), we deduce that

$$
\begin{aligned}
& \frac{1}{2} \frac{d}{d t}\left(\|v\|_{H^{2}}^{2}+\|\nabla \psi\|_{H^{2}}^{2}\right)+\|\nabla v\|_{H^{2}}^{2} \\
= & -(v \cdot \nabla v \mid v)_{H^{2}}+(\Delta \psi \mid v \cdot \nabla \psi)_{H^{2}}-(\operatorname{div}(\nabla \psi \otimes \nabla \psi) \mid v)_{H^{2}} .
\end{aligned}
$$

Next we take the standard $H^{1}$ inner product of $(2.6)_{3}$ with $\Delta \psi$, using again the integration by parts, to obtain

$$
\left(\partial_{t} v_{3} \mid \Delta \psi\right)_{H^{1}}+\left(v \cdot \nabla v_{3} \mid \Delta \psi\right)_{H^{1}}-\left(\Delta v_{3} \mid \Delta \psi\right)_{H^{1}}=-\left\|\nabla_{h} \nabla \psi\right\|_{H^{1}}^{2}+\left(f^{v} \mid \Delta \psi\right)_{H^{1}} .
$$

From the equation (1.2) 1 and again the integration by parts, we get

$$
\begin{aligned}
& \left(\partial_{t} v_{3} \mid \Delta \psi\right)_{H^{1}} \\
= & \frac{d}{d t}\left(v_{3} \mid \Delta \psi\right)_{H^{1}}-\left(v_{3} \mid \Delta \partial_{t} \psi\right)_{H^{1}} \\
= & \frac{d}{d t}\left(v_{3} \mid \Delta \psi\right)_{H^{1}}+\left(v_{3} \mid \Delta\left(v \cdot \nabla \psi+v_{3}\right)\right)_{H^{1}} \\
= & \frac{d}{d t}\left(v_{3} \mid \Delta \psi\right)_{H^{1}}-\left(\nabla v_{3} \mid \nabla(v \cdot \nabla \psi)\right)_{H^{1}}-\left\|\nabla v_{3}\right\|_{H^{1}}^{2} .
\end{aligned}
$$

We observe, by (1.2) 1 , that

$$
\begin{aligned}
& -\left(\Delta v_{3} \mid \Delta \psi\right)_{H^{1}} \\
= & \left(\Delta\left(\partial_{t} \psi+v \cdot \nabla \psi\right) \mid \Delta \psi\right)_{H^{1}} \\
= & \frac{1}{2} \frac{d}{d t}\|\Delta \psi\|_{H^{1}}^{2}+(\Delta(v \cdot \nabla \psi) \mid \Delta \psi)_{H^{1}} .
\end{aligned}
$$

Combining (2.12)-(2.14), we hence conclude

$$
\begin{aligned}
& \frac{d}{d t}\left\{\frac{1}{2}\|\Delta \psi\|_{H^{1}}^{2}+\left(v_{3} \mid \Delta \psi\right)_{H^{1}}\right\}+\left\|\nabla_{h} \nabla \psi\right\|_{H^{1}}^{2}-\left\|\nabla v_{3}\right\|_{H^{1}}^{2} \\
= & -\left(v \cdot \nabla v_{3} \mid \Delta \psi\right)_{H^{1}}+\left(f^{v} \mid \Delta \psi\right)_{H^{1}}+\left(\nabla v_{3} \mid \nabla(v \cdot \nabla \psi)\right)_{H^{1}}-(\Delta(v \cdot \nabla \psi) \mid \Delta \psi) .
\end{aligned}
$$

With (2.11) and (2.15), one can complete the proof.

The following is the key a priori estimate which is essential to the proof of the main result of this paper.

Lemma 2.3. Let $(\psi, v)$ be sufficiently smooth functions which solve (1.2) and satisfy $\nabla \psi \in L_{T}^{\infty}\left(H^{2}\left(\mathbb{R}^{3}\right)\right)$, $\nabla_{h} \nabla \psi \in L_{T}^{2}\left(H^{1}\left(\mathbb{R}^{3}\right)\right), v \in L_{T}^{\infty}\left(H^{2}\left(\mathbb{R}^{3}\right)\right)$ and $\nabla v \in L_{T}^{2}\left(H^{2}\left(\mathbb{R}^{3}\right)\right)$, then there holds

$$
B_{T}^{2} \leq C\left(\left\|v_{0}\right\|_{H^{2}\left(\mathbb{R}^{3}\right)}^{2}+\left\|\nabla \psi_{0}\right\|_{H^{2}\left(\mathbb{R}^{3}\right)}^{2}\right)+C B_{T}^{3}\left(1+B_{T}\right)^{2} .
$$

where $C$ is a positive constant independent of $T$.

Proof. By the energy estimate (2.7) and the definition of $B_{T}$, we get for a positive constant $C$ (independent of $T$ ) that

$$
\begin{aligned}
B_{T}^{2} \leq & C B_{0}^{2}+C\left|\int_{0}^{T}(v \cdot \nabla v \mid v)_{H^{2}} d t\right|+C\left|\int_{0}^{T}(v \cdot \nabla \psi \mid \Delta \psi)_{H^{2}} d t\right| \\
& +C\left|\int_{0}^{T}(\operatorname{div}(\nabla \psi \otimes \nabla \psi) \mid v)_{H^{2}} d t\right|+C\left|\int_{0}^{T}\left(v \cdot \nabla v_{3} \mid \Delta \psi\right)_{H^{1}} d t\right|+C\left|\int_{0}^{T}\left(f^{v} \mid \Delta \psi\right)_{H^{1}} d t\right| \\
& +C\left|\int_{0}^{T}\left(\nabla v_{3} \mid \nabla(v \cdot \nabla \psi)\right)_{H^{1}} d t\right|+C\left|\int_{0}^{T}(\Delta(v \cdot \nabla \psi) \mid \Delta \psi)_{H^{1}} d t\right|
\end{aligned}
$$




$$
:=C B_{0}^{2}+\sum_{j=1}^{7} I_{j} .
$$

We are going to estimate term by term the right hand side of the inequality. The basic strategies involved in estimating all such quantities are the same. More precisely, we estimate separately terms involving horizontal derivatives and terms with vertical derivatives. For terms with horizontal derivatives $\nabla_{h} \psi$, one can use the dissipations implied by the energy equality (2.7). For terms containing vertical derivatives, we use the algebriac relation (deduced from that $\operatorname{div} v=0$ ) and the transport equations. The latter reduces space-time estimates to bounds on time-slices and terms with either horizontal derivatives or of higher order nonlinearities (hence they are smaller under our smallness assumptions on the initial data). To illustrate the basic idea, we start with the second term $I_{2}$. Applying the Gagliardo-NirenbergSobolev type estimates in Lemma 2.1. and use the fact that $\operatorname{div} v=0$, Hölder and Sobolev inequalities, we deduce that

$$
\begin{aligned}
& I_{2}=C\left|\int_{0}^{T}(v \cdot \nabla \psi \mid \Delta \psi)_{H^{2}} d t\right| \\
= & C\left|\sum_{|\alpha| \leq 2} \sum_{i=1}^{3} \int_{0}^{T} \int\left[\partial^{\alpha} \partial_{i}(v \cdot \nabla \psi)-v \cdot \nabla \partial^{\alpha} \partial_{i} \psi\right] \partial^{\alpha} \partial_{i} \psi d x d t\right| \\
\leq & C\|\nabla v\|_{L_{T}^{2}\left(L^{2}\left(\mathbb{R}^{3}\right)\right)}\|\nabla \psi\|_{L_{T}^{4}\left(L^{4}\left(\mathbb{R}^{3}\right)\right)}^{2}+C\left\|\nabla^{2} \psi\right\|_{L_{T}^{4}\left(L^{4}\left(\mathbb{R}^{3}\right)\right)}\left(\left\|\nabla^{2} v\right\|_{L_{T}^{2}\left(L^{2}\left(\mathbb{R}^{3}\right)\right)}\|\nabla \psi\|_{L_{T}^{4}\left(L^{4}\left(\mathbb{R}^{3}\right)\right)}\right. \\
& \left.+\|\nabla v\|_{L_{T}^{2}\left(L^{2}\left(\mathbb{R}^{3}\right)\right)}\left\|\nabla^{2} \psi\right\|_{L_{T}^{4}\left(L^{4}\left(\mathbb{R}^{3}\right)\right)}\right)+C\left\|\nabla_{h} \nabla^{2} \psi\right\|_{L_{T}^{2}\left(L^{2}\left(\mathbb{R}^{3}\right)\right)}\left(\left\|\nabla^{3} v\right\|_{L_{T}^{2}\left(L^{2}\left(\mathbb{R}^{3}\right)\right)}\|\nabla \psi\|_{L_{T}^{\infty}\left(L^{\infty}\left(\mathbb{R}^{3}\right)\right)}\right. \\
& \left.+\left\|\nabla^{2} v\right\|_{L_{T}^{2}\left(L^{4}\left(\mathbb{R}^{3}\right)\right)}\left\|\nabla^{2} \psi\right\|_{L_{T}^{\infty}\left(L^{4}\left(\mathbb{R}^{3}\right)\right)}+\|\nabla v\|_{L_{T}^{2}\left(L^{\infty}\left(\mathbb{R}^{3}\right)\right)}\left\|\nabla^{3} \psi\right\|_{L_{T}^{\infty}\left(L^{2}\left(\mathbb{R}^{3}\right)\right)}\right) \\
& +C\left\|\nabla^{3} \psi\right\|_{L_{T}^{\infty}\left(L^{2}\left(\mathbb{R}^{3}\right)\right)}\left(\left\|\nabla^{3} v_{h}\right\|_{L_{T}^{2}\left(L^{2}\left(\mathbb{R}^{3}\right)\right)}\left\|\nabla_{h} \psi\right\|_{L_{T}^{2}\left(L^{\infty}\left(\mathbb{R}^{3}\right)\right)}+\left\|\nabla^{2} v_{h}\right\|_{L_{T}^{2}\left(L^{4}\left(\mathbb{R}^{3}\right)\right)}\left\|\nabla \nabla_{h} \psi\right\|_{L_{T}^{2}\left(L^{4}\left(\mathbb{R}^{3}\right)\right)}\right. \\
& \left.+\left\|\nabla v_{h}\right\|_{L_{T}^{2}\left(L^{\infty}\left(\mathbb{R}^{3}\right)\right)}\left\|\nabla^{2} \nabla_{h} \psi\right\|_{L_{T}^{2}\left(L^{2}\left(\mathbb{R}^{3}\right)\right)}\right) \\
& +C\left|\int_{0}^{T} \int \partial_{3}^{3} \psi\left(\partial_{3}^{3} v_{3} \partial_{3} \psi+3 \partial_{3}^{2} v_{3} \partial_{3}^{2} \psi+3 \partial_{3} v_{3} \partial_{3}^{3} \psi\right) d x d t\right|
\end{aligned}
$$

To estimate the last term in the above inequality (2.18), we need the following two technical lemmas. The proofs of these two Lemmas will be given in the Appendix.

Lemma 2.4. Under the conditions in Lemma 2.3, then there holds

$$
\begin{aligned}
& \left|\int_{0}^{T} \int_{\mathbb{R}^{3}} \partial_{3} \psi \partial_{3}^{3} \psi \partial_{3}^{3} v_{3} d x d t\right|+\left|\int_{0}^{T} \int_{\mathbb{R}^{3}} \partial_{3}^{2} \psi \partial_{3}^{3} \psi \partial_{3}^{2} v_{3} d x d t\right|+\left|\int_{0}^{T} \int_{\mathbb{R}^{3}} \partial_{3} \psi \partial_{3}^{3} \psi \partial_{3}^{2} v_{3} \partial_{3}^{2} \psi d x d t\right| \\
\leq & C\|\nabla \psi\|_{L_{T}^{\infty}\left(H^{2}\left(\mathbb{R}^{3}\right)\right)}\left\|\nabla_{h} \nabla \psi\right\|_{L_{T}^{2}\left(H^{1}\left(\mathbb{R}^{3}\right)\right)}\|\nabla v\|_{L_{T}^{2}\left(H^{2}\left(\mathbb{R}^{3}\right)\right)}\left(1+\|\nabla \psi\|_{L_{T}^{\infty}\left(H^{2}\left(\mathbb{R}^{3}\right)\right)}\right),
\end{aligned}
$$

where $C$ is a positive constant independent of $T$.

Lemma 2.5. Under the conditions in Lemma 2.3, then there holds

$$
\left|\int_{0}^{T} \int_{\mathbb{R}^{3}} \partial_{3} v_{3}\left(\partial_{3}^{3} \psi\right)^{2} d x d t\right| \leq C B_{T}^{3}\left(1+B_{T}^{2}\right),
$$

where $C$ is a positive constant independent of $T$.

Accepting these two lemmas, we proceed with our proof of the key estimate in Lemma 2.3. By (2.18) and Lemmas 2.4]2.5, we obtain

$$
I_{2}=C\left|\int_{0}^{T}(v \cdot \nabla \psi \mid \Delta \psi)_{H^{2}} d t\right| \leq C B_{T}^{3}\left(1+B_{T}\right)^{2} .
$$


Similarly, one can estimate that

$$
\begin{aligned}
I_{7} & =C\left|\int_{0}^{T}(\Delta(v \cdot \nabla \psi) \mid \Delta \psi)_{H^{1}} d t\right| \\
& =C\left|\sum_{i=1}^{3} \int_{0}^{T}\left(\partial_{i}(v \cdot \nabla \psi) \mid \partial_{i} \Delta \psi\right)_{H^{1}} d t\right| \\
& \leq C B_{T}^{3}\left(1+B_{T}\right)^{2} .
\end{aligned}
$$

Next, we estimate $I_{3}$ as follows:

$$
\begin{aligned}
I_{3}= & C\left|\int_{0}^{T}(\operatorname{div}(\nabla \psi \otimes \nabla \psi) \mid v)_{H^{2}} d t\right| \\
= & C\left|\sum_{|\alpha| \leq 2} \sum_{i, j=1}^{3} \int_{0}^{T} \int \partial^{\alpha}\left(\partial_{i} \psi \partial_{j} \psi\right) \partial^{\alpha} \partial_{i} v_{j} d x d t\right| \\
\leq & C\|\nabla v\|_{L_{T}^{2}\left(L^{2}\left(\mathbb{R}^{3}\right)\right)} \mid\|\nabla \psi\|_{L_{T}^{4}\left(L^{4}\left(\mathbb{R}^{3}\right)\right)}^{2}+C\left\|\nabla^{2} v\right\|_{L_{T}^{2}\left(L^{2}\left(\mathbb{R}^{3}\right)\right)}\|\nabla \psi\|_{L_{T}^{4}\left(L^{4}\left(\mathbb{R}^{3}\right)\right)}\left\|\nabla^{2} \psi\right\|_{L_{T}^{4}\left(L^{4}\left(\mathbb{R}^{3}\right)\right)} \\
& +C\left\|\nabla^{3} v\right\|_{L_{T}^{2}\left(L^{2}\left(\mathbb{R}^{3}\right)\right)}\left(\left\|\nabla^{2} \psi\right\|_{L_{T}^{4}\left(L^{4}\left(\mathbb{R}^{3}\right)\right)}^{2}+\left\|\nabla_{h} \psi\right\|_{L_{T}^{2}\left(L^{\infty}\left(\mathbb{R}^{3}\right)\right)}\left\|\nabla^{3} \psi\right\|_{L_{T}^{\infty}\left(L^{2}\left(\mathbb{R}^{3}\right)\right)}\right. \\
& \left.+\|\nabla \psi\|_{L_{T}^{\infty}\left(L^{\infty}\left(\mathbb{R}^{3}\right)\right)}\left\|\nabla^{2} \nabla_{h} \psi\right\|_{L_{T}^{2}\left(L^{2}\left(\mathbb{R}^{3}\right)\right)}\right)+C\left|\int_{0}^{T} \int \partial_{3}^{3} v_{3} \partial_{3} \psi \partial_{3}^{3} \psi d x d t\right| \\
\leq & C B_{T}^{3} .
\end{aligned}
$$

Apply the same line of arguments, one can deduce that

$$
\begin{aligned}
& \left|\sum_{i, j=1}^{3} \int_{0}^{T}\left(\partial_{3}(-\Delta)^{-1}\left(\partial_{i} v_{j} \partial_{j} v_{i}\right) \mid \Delta \psi\right)_{H^{1}} d t\right| \\
\leq & C \int_{0}^{T}\left\|(\nabla v)^{2}\right\|_{H^{1}\left(\mathbb{R}^{3}\right)}\|\nabla \psi\|_{H^{1}\left(\mathbb{R}^{3}\right)} d t \\
\leq & C\|\nabla v\|_{L_{T}^{2}\left(H^{2}\left(\mathbb{R}^{3}\right)\right)}^{2}\|\nabla \psi\|_{L_{T}^{\infty}\left(H^{2}\left(\mathbb{R}^{3}\right)\right)} .
\end{aligned}
$$

Similarly, one has

$$
\begin{aligned}
& \left|\int_{0}^{T}\left(-\sum_{i, j=1}^{3} \partial_{3}(-\Delta)^{-1}\left(\partial_{i} \partial_{j}\left(\partial_{i} \psi \partial_{j} \psi\right)\right)-\sum_{j=1}^{3} \partial_{j}\left(\partial_{3} \psi \partial_{j} \psi\right) \mid \Delta \psi\right)_{H^{1}} d t\right| \\
= & \left|\int_{0}^{T}\left(-\sum_{i, j=1}^{2} \partial_{3}(-\Delta)^{-1}\left(\partial_{i} \partial_{j}\left(\partial_{i} \psi \partial_{j} \psi\right)\right) \mid \Delta \psi\right)_{H^{1}} d t\right| \\
& +\left|\int_{0}^{T} \sum_{j=1}^{2}\left(2 \partial_{3}(-\Delta)^{-1}\left(\partial_{3}\left(\partial_{3} \psi \partial_{j} \psi\right)\right)+\left(\partial_{3} \psi \partial_{j} \psi\right) \mid \partial_{j} \Delta \psi\right)_{H^{1}} d t\right| \\
& +\left|\int_{0}^{T}\left(-(-\Delta)^{-1} \partial_{3}^{3}\left(\partial_{3} \psi\right)^{2}-\partial_{3}\left(\partial_{3} \psi\right)^{2} \mid \Delta \psi\right)_{H^{1}} d t\right| \\
\leq & C \int_{0}^{T}\left\|\nabla\left(\nabla_{h} \psi\right)^{2}\right\|_{H^{1}\left(\mathbb{R}^{3}\right)}\|\Delta \psi\|_{H^{1}\left(\mathbb{R}^{3}\right)} d t+C \int_{0}^{T}\left\|\nabla\left(\nabla \psi \nabla_{h} \psi\right)\right\|_{H^{1}\left(\mathbb{R}^{3}\right)}\left\|\nabla \nabla_{h} \psi\right\|_{H^{1}\left(\mathbb{R}^{3}\right)} d t \\
& +\left|\int_{0}^{T}\left(\sum_{i=1}^{2}(-\Delta)^{-1} \partial_{3} \partial_{i}^{2}\left(\partial_{3} \psi\right)^{2} \mid \Delta \psi\right)_{H^{1}} d t\right| \\
\leq & C\left\|\nabla \nabla_{h} \psi\right\|_{L_{T}^{2}\left(H^{1}\left(\mathbb{R}^{3}\right)\right)}^{2}\|\nabla \psi\|_{L_{T}^{\infty}\left(H^{2}\left(\mathbb{R}^{3}\right)\right)}+C \int_{0}^{T}\left\|\nabla_{h}(\nabla \psi)^{2}\right\|_{H^{1}\left(\mathbb{R}^{3}\right)}\left\|\nabla \nabla_{h} \psi\right\|_{H^{1}\left(\mathbb{R}^{3}\right)} d t
\end{aligned}
$$




$$
\leq \quad C\left\|\nabla \nabla_{h} \psi\right\|_{L_{T}^{2}\left(H^{1}\left(\mathbb{R}^{3}\right)\right)}^{2}\|\nabla \psi\|_{L_{T}^{\infty}\left(H^{2}\left(\mathbb{R}^{3}\right)\right)} .
$$

Combining (2.24)-(2.25), one concludes

$$
I_{5}=C\left|\int_{0}^{T}\left(f^{v} \mid \Delta \psi\right)_{H^{1}} d t\right| \leq C B_{T}^{3} .
$$

One can obtain the following estimates in the same way, to save the ink, we omit the details.

$$
\begin{gathered}
I_{1}=C\left|\int_{0}^{T}(v \cdot \nabla v \mid v)_{H^{2}} d t\right| \leq C\|\nabla v\|_{L_{T}^{2}\left(H^{2}\left(\mathbb{R}^{3}\right)\right)}^{2}\|v\|_{L_{T}^{\infty}\left(H^{2}\left(\mathbb{R}^{3}\right)\right)}, \\
I_{4}=C\left|\int_{0}^{T}\left(v \cdot \nabla v_{3} \mid \Delta \psi\right)_{H^{1}} d t\right| \leq C\|\nabla v\|_{L_{T}^{2}\left(H^{2}\left(\mathbb{R}^{3}\right)\right)}^{2}\|\nabla \psi\|_{L_{T}^{\infty}\left(H^{2}\left(\mathbb{R}^{3}\right)\right)}, \\
I_{6}=C\left|\int_{0}^{T}\left(\nabla v_{3} \mid \nabla(v \cdot \nabla \psi)\right)_{H^{1}} d t\right| \leq C\|\nabla v\|_{L_{T}^{2}\left(H^{2}\left(\mathbb{R}^{3}\right)\right)}^{2}\|\nabla \psi\|_{L_{T}^{\infty}\left(H^{2}\left(\mathbb{R}^{3}\right)\right)},
\end{gathered}
$$

Summing up (2.17), (2.21)-(2.23) and (2.26)-(2.29), we conclude (2.16).

Remark 2.1. In several places of our proofs, we have used the fact that

$$
\left\|\nabla_{h} \psi\right\|_{L_{T}^{2}\left(L^{\infty}\left(\mathbb{R}^{3}\right)\right)} \leq C\left\|\nabla_{h} \psi\right\|_{L_{T}^{2}\left(W^{1,6}\left(\mathbb{R}^{3}\right)\right)} \leq C\left\|\nabla \nabla_{h} \psi\right\|_{L_{T}^{2}\left(H^{1}\left(\mathbb{R}^{3}\right)\right)},
$$

which is a direct consequence of Sobolev embedding Theorem. When the spatial dimension is two, we cannot use $\left\|\nabla \nabla_{h} \psi\right\|_{L_{T}^{2}\left(H^{1}\left(\mathbb{R}^{2}\right)\right)}$ to bound $\left\|\nabla_{h} \psi\right\|_{L_{T}^{2}\left(L^{\infty}\left(\mathbb{R}^{2}\right)\right)}$. So it is necessary to make various changes in order for the proofs in this article to work in the case that the spatial dimension is two. On the other hand, if one assume that the initial data are in $H^{2} \times \dot{H}^{-s}\left(\mathbb{R}^{2}\right), s \in\left(\frac{1}{2}, 1\right)$, then use $\left\|\nabla^{1+s} \nabla_{h} \psi\right\|_{L_{T}^{2}\left(H^{1}\left(\mathbb{R}^{2}\right)\right)}$ to bound $\left\|\nabla_{h} \psi\right\|_{L_{T}^{2}\left(L^{\infty}\left(\mathbb{R}^{2}\right)\right)}$ and obtain the similar result though the arguments are technically more complicated.

\section{Proof of Theorem 1.1}

Via the analysis in [15], one can get the following local existence result following now the standard arguments:

Theorem 3.1. Assume that the initial data $\left(\psi_{0}, v_{0}\right)$ satisfy $\left(\nabla \psi_{0}, v_{0}\right) \in H^{2}\left(\mathbb{R}^{3}\right) \times H^{2}\left(\mathbb{R}^{3}\right)$, then there exists $T_{0}>0$ such that the system (1.2) has a unique local solution $(\psi, v, \nabla p)$ on $\left[0, T_{0}\right]$ satisfying

$$
\begin{gathered}
\nabla \psi, v \in C\left(\left[0, T_{0}\right] ; H^{2}\left(\mathbb{R}^{3}\right)\right), \nabla v \in L^{2}\left(\left[0, T_{0}\right] ; H^{2}\left(\mathbb{R}^{3}\right)\right), \\
\nabla p \in L^{\infty}\left(\left[0, T_{0}\right] ; H^{1}\left(\mathbb{R}^{3}\right)\right) .
\end{gathered}
$$

Proof of Theorem 1.1. Theorem 3.1 implies that the system (1.2) has a unique local strong solution $(\psi, v, \nabla p)$ on $\left[0, T^{*}\right)$, where $\left[0, T^{*}\right)$ is the maximal existence time interval for the above solution. Our goal is to prove $T^{*}=\infty$ provided that the initial data $\left(\psi_{0}, v_{0}\right)$ satisfy (1.5).

Assume that $(\psi, v, \nabla p)$ is the unique local strong solution of (1.2) on $\left[0, T^{*}\right)$, and satisfies (3.1)-(3.2). From (2.16), we have

$$
B_{T}^{2} \leq C\left(\left\|v_{0}\right\|_{H^{2}\left(\mathbb{R}^{3}\right)}^{2}+\left\|\nabla \psi_{0}\right\|_{H^{2}\left(\mathbb{R}^{3}\right)}^{2}\right)+C B_{T}^{3}\left(1+B_{T}\right)^{2},
$$

for all $T \in\left(0, T^{*}\right)$. If the initial data $\left(\psi_{0}, v_{0}\right)$ satisfy (1.5), where $c_{0}$ satisfies

$$
C \sqrt{2 C} c_{0}\left(1+\sqrt{2 C} c_{0}\right)^{2} \leq \frac{1}{2}
$$

then one can easily obtain

$$
B_{T}^{2} \leq 2 C B_{0}^{2}, \text { for all } T \in\left(0, T^{*}\right)
$$

As the right hand side of the last inequality above remains to be small, we must have that $T^{*}=\infty$, and hence (1.6) holds. From (2.5), we see that $\nabla p \in L^{\infty}\left([0, \infty) ; H^{1}\right)$ and (1.7) holds. This finishes the proof of Theorem 1.1. 


\section{Appendix}

Here we shall give the proofs of two technical Lemmas 2.4 2.5 that are needed in establishing the key $a$ priori estimates. As $\operatorname{div} v=0$, we can replace $\operatorname{div}_{h} v_{h}=\partial_{1} v_{1}+\partial_{2} v_{2}$ by $\partial_{3} v_{3}$ in various calculations in the proof of Lemma 2.4. Sometime, it would be useful (and it may be also necessary) to replace $v_{3}$. In fact, via $(1.2)$, we can re-write

$$
v_{3}=-\left(\partial_{t} \psi+v \cdot \nabla \psi\right) .
$$

The above substitution for $v_{3}$ has the advantage that it reduces space-time integral estimates to estimates on time slices and space times integral with higher order nonlinearities and fast dissipation. The latter is smaller by the initial smallness assumptions.

Proof of Lemma 2.4. Using the integration by parts, the fact that $\operatorname{div} v=0$, the Hölder's inequality and the Sobolev embedding Theorem, we can estimate the first term in the lemma 2.4 as follows:

$$
\begin{aligned}
& \left|\int_{0}^{T} \int \partial_{3} \psi \partial_{3}^{3} \psi \partial_{3}^{3} v_{3} d x d t\right| \\
= & \left|\int_{0}^{T} \int \partial_{3} \psi \partial_{3}^{3} \psi \partial_{3}^{2} \operatorname{div}_{h} v_{h} d x d t\right| \\
= & \left|\int_{0}^{T} \int\left(-\partial_{3}^{2} v_{h} \cdot \nabla_{h} \partial_{3} \psi \partial_{3}^{3} \psi-\partial_{3} \psi \partial_{3}^{2} v_{h} \cdot \nabla_{h} \partial_{3}^{3} \psi\right) d x d t\right| \\
= & \left|\int_{0}^{T} \int\left(-\partial_{3}^{2} v_{h} \cdot \nabla_{h} \partial_{3} \psi \partial_{3}^{3} \psi+\partial_{3}^{2} \psi \partial_{3}^{2} v_{h} \cdot \nabla_{h} \partial_{3}^{2} \psi+\partial_{3} \psi \partial_{3}^{3} v_{h} \cdot \nabla_{h} \partial_{3}^{2} \psi\right) d x d t\right| \\
\leq & C\left\|\partial_{3}^{2} v_{h}\right\|_{L_{T}^{2}\left(L^{4}\left(\mathbb{R}^{3}\right)\right)}\left\|\nabla_{h} \partial_{3} \psi\right\|_{L_{T}^{2}\left(L^{4}\left(\mathbb{R}^{3}\right)\right)}\left\|\partial_{3}^{3} \psi\right\|_{L_{T}^{\infty}\left(L^{2}\left(\mathbb{R}^{3}\right)\right)} \\
& +C\left\|\partial_{3}^{2} \psi\right\|_{L_{T}^{\infty}\left(L^{4}\left(\mathbb{R}^{3}\right)\right)}\left\|\partial_{3}^{2} v_{h}\right\|_{L_{T}^{2}\left(L^{4}\left(\mathbb{R}^{3}\right)\right)}\left\|\nabla_{h} \partial_{3}^{2} \psi\right\|_{L_{T}^{2}\left(L^{2}\left(\mathbb{R}^{3}\right)\right)} \\
& +C\left\|\partial_{3} \psi\right\|_{L_{T}^{\infty}\left(L^{\infty}\left(\mathbb{R}^{3}\right)\right)}\left\|\partial_{3}^{3} v_{h}\right\|_{L_{T}^{2}\left(L^{2}\left(\mathbb{R}^{3}\right)\right)}\left\|\nabla_{h} \partial_{3}^{2} \psi\right\|_{L_{T}^{2}\left(L^{2}\left(\mathbb{R}^{3}\right)\right)} \\
\leq & C\|\nabla \psi\|_{L_{T}^{\infty}\left(H^{2}\left(\mathbb{R}^{3}\right)\right)}\left\|\nabla_{h} \nabla \psi\right\|_{L_{T}^{2}\left(H^{1}\left(\mathbb{R}^{3}\right)\right)}\|\nabla v\|_{L_{T}^{2}\left(H^{2}\left(\mathbb{R}^{3}\right)\right)}
\end{aligned}
$$

The other terms in (2.19) can be treated similarly, and we can conclude Lemma 2.4 .

We shall now proceed with the proof lemma 2.5. The basic strategy has been described earlier, see for example, (1.9) . For this purpose, we first prove the following lemma. Here we use the equation (1.2) 1 to bounded the term $\int_{0}^{T} \int \partial_{3} \psi \partial_{3} v_{3}\left(\partial_{3}^{3} \psi\right)^{2} d x d t$.

Lemma 3.1. Under the conditions of Lemma 2.3, then there holds

$$
\left|\int_{0}^{T} \int \partial_{3} \psi \partial_{3} v_{3}\left(\partial_{3}^{3} \psi\right)^{2} d x d t\right| \leq C B_{T}^{4}\left(1+B_{T}\right),
$$

where $C$ is a positive constant independent of $T$.

Proof. Applying (2.3), (3.6), the integration by parts, Hölder's inequality and Sobolev embedding Theorem, we get

$$
\begin{aligned}
& \left|\int_{0}^{T} \int \partial_{3} \psi \partial_{3} v_{3}\left(\partial_{3}^{3} \psi\right)^{2} d x d t\right| \\
= & \left|\int_{0}^{T} \int \partial_{3} \psi \partial_{3}\left(\partial_{t} \psi+v \cdot \nabla \psi\right)\left(\partial_{3}^{3} \psi\right)^{2} d x d t\right| \\
= & \left|\int \frac{1}{2}\left(\partial_{3} \psi\right)^{2}\left(\partial_{3}^{3} \psi\right)^{2} d x\right|_{0}^{T}+\int_{0}^{T} \int\left[-\left(\partial_{3} \psi\right)^{2} \partial_{3}^{3} \psi \partial_{3}^{3} \partial_{t} \psi+\partial_{3} \psi \partial_{3}(v \cdot \nabla \psi)\left(\partial_{3}^{3} \psi\right)^{2}\right] d x d t \mid \\
\leq & C\left\|\partial_{3} \psi\right\|_{L_{T}^{\infty}\left(L^{\infty}\left(\mathbb{R}^{3}\right)\right)}^{2}\left\|\partial_{3}^{3} \psi\right\|_{L_{T}^{\infty}\left(L^{2}\left(\mathbb{R}^{3}\right)\right)}^{2}
\end{aligned}
$$




$$
\begin{aligned}
& +\left|\int_{0}^{T} \int\left[\left(\partial_{3} \psi\right)^{2} \partial_{3}^{3} \psi \partial_{3}^{3}\left(v \cdot \nabla \psi+v_{3}\right)+\partial_{3} \psi \partial_{3}(v \cdot \nabla \psi)\left(\partial_{3}^{3} \psi\right)^{2}\right] d x d t\right| \\
\leq & C\|\nabla \psi\|_{L_{T}^{\infty}\left(H^{2}\left(\mathbb{R}^{3}\right)\right)}^{4}+C\left\|\partial_{3} \psi\right\|_{L_{T}^{4}\left(L^{\infty}\left(\mathbb{R}^{3}\right)\right)}^{2}\left\|\partial_{3}^{3} \psi\right\|_{L_{T}^{\infty}\left(L^{2}\left(\mathbb{R}^{3}\right)\right)}\left\|\partial_{3}^{3}(v \cdot \nabla \psi)-v \cdot \nabla \partial_{3}^{3} \psi\right\|_{L_{T}^{2}\left(L^{2}\left(\mathbb{R}^{3}\right)\right)} \\
& +C\left\|\partial_{3} \psi\right\|_{L_{T}^{4}\left(L^{\infty}\left(\mathbb{R}^{3}\right)\right)}^{2}\left\|\partial_{3}^{3} \psi\right\|_{L_{T}^{\infty}\left(L^{2}\left(\mathbb{R}^{3}\right)\right)}\left\|\partial_{3}^{3} v_{3}\right\|_{L_{T}^{2}\left(L^{2}\left(\mathbb{R}^{3}\right)\right)} \\
& +\left|\int_{0}^{T} \int\left\{\frac{1}{2}\left[\left(\partial_{3} \psi\right)^{2} v \cdot \nabla\left(\partial_{3}^{3} \psi\right)^{2}+v \cdot \nabla\left(\partial_{3} \psi\right)^{2}\left(\partial_{3}^{3} \psi\right)^{2}\right]\right\} d x d t\right| \\
& +\left|\int_{0}^{T} \int\left\{\partial_{3} \psi \partial_{3} v \cdot \nabla \psi\left(\partial_{3}^{3} \psi\right)^{2}\right\} d x d t\right| \\
\leq & C B_{T}^{4}\left(1+B_{T}\right) .
\end{aligned}
$$

In deriving the last inequality above, we have used the following calculations:

$$
\begin{aligned}
& \left\|\partial_{3}^{3}(v \cdot \nabla \psi)-v \cdot \nabla \partial_{3}^{3} \psi\right\|_{L_{T}^{2}\left(L^{2}\left(\mathbb{R}^{3}\right)\right)} \\
\leq & C\left\|\nabla^{3} v\right\|_{L_{T}^{2}\left(L^{2}\left(\mathbb{R}^{3}\right)\right)}\|\nabla \psi\|_{L_{T}^{\infty}\left(L^{\infty}\left(\mathbb{R}^{3}\right)\right)}+C\left\|\nabla^{2} v\right\|_{L_{T}^{2}\left(L^{4}\left(\mathbb{R}^{3}\right)\right)}\left\|\nabla^{2} \psi\right\|_{L_{T}^{\infty}\left(L^{4}\left(\mathbb{R}^{3}\right)\right)} \\
& +C\|\nabla v\|_{L_{T}^{2}\left(L^{\infty}\left(\mathbb{R}^{3}\right)\right)}\left\|\nabla^{3} \psi\right\|_{L_{T}^{\infty}\left(L^{2}\left(\mathbb{R}^{3}\right)\right)} \\
\leq & C\|\nabla v\|_{L_{T}^{2}\left(H^{2}\left(\mathbb{R}^{3}\right)\right)}\|\nabla \psi\|_{L_{T}^{\infty}\left(H^{2}\left(\mathbb{R}^{3}\right)\right)},
\end{aligned}
$$

and also the estimation:

$$
\begin{aligned}
& \left|\int_{0}^{T} \int\left\{\partial_{3} \psi \partial_{3} v \cdot \nabla \psi\left(\partial_{3}^{3} \psi\right)^{2}\right\} d x d t\right| \\
\leq & \|\nabla \psi\|_{L_{T}^{4}\left(L^{\infty}\left(\mathbb{R}^{3}\right)\right)}^{2}\|\nabla v\|_{L_{T}^{2}\left(L^{\infty}\left(\mathbb{R}^{3}\right)\right)}\left\|\nabla^{3} \psi\right\|_{L_{T}^{\infty}\left(L^{2}\left(\mathbb{R}^{3}\right)\right)}^{2} .
\end{aligned}
$$

Proof of Lemma 2.5. We use Lemma 2.1, (2.19), (3.6), (3.7), the integration by parts, Hölder's inequality and Sobolev embedding Theorem to do following derivations:

$$
\begin{aligned}
& \left|\int_{0}^{T} \int \partial_{3} v_{3}\left(\partial_{3}^{3} \psi\right)^{2} d x d t\right| \\
= & \left|\int_{0}^{T} \int \partial_{3}\left(\partial_{t} \psi+v \cdot \nabla \psi\right)\left(\partial_{3}^{3} \psi\right)^{2} d x d t\right| \\
= & \left|\int \partial_{3} \psi\left(\partial_{3}^{3} \psi\right)^{2} d x\right|_{0}^{T}+\int_{0}^{T} \int\left\{-2 \partial_{3} \psi \partial_{3}^{3} \psi \partial_{3}^{3} \partial_{t} \psi+\partial_{3}(v \cdot \nabla \psi)\left(\partial_{3}^{3} \psi\right)^{2}\right\} d x d t \mid \\
\leq & C\left\|\partial_{3} \psi\right\|_{L_{T}^{\infty}\left(L^{\infty}\left(\mathbb{R}^{3}\right)\right)}\left\|\partial_{3}^{3} \psi\right\|_{L_{T}^{\infty}\left(L^{2}\left(\mathbb{R}^{3}\right)\right)}^{2} \\
& +\left|\int_{0}^{T} \int\left\{2 \partial_{3} \psi \partial_{3}^{3} \psi \partial_{3}^{3}\left(v \cdot \nabla \psi+v_{3}\right)+\partial_{3}(v \cdot \nabla \psi)\left(\partial_{3}^{3} \psi\right)^{2}\right\} d x d t\right| \\
\leq & C\|\nabla \psi\|_{L_{T}^{\infty}\left(H^{2}\left(\mathbb{R}^{3}\right)\right)}^{3}+C\|\nabla \psi\|_{L_{T}^{\infty}\left(H^{2}\left(\mathbb{R}^{3}\right)\right)}\left\|\nabla \nabla_{h} \psi\right\|_{L_{T}^{2}\left(H^{1}\left(\mathbb{R}^{3}\right)\right)}\|\nabla v\|_{L_{T}^{2}\left(H^{2}\left(\mathbb{R}^{3}\right)\right)} \\
& +\mid \int_{0}^{T} \int\left\{2 \partial_{3} \psi \partial_{3}^{3} \psi\left[\partial_{3}^{3}(v \cdot \nabla \psi)-v \cdot \nabla \partial_{3}^{3} \psi\right]+\partial_{3} \psi v \cdot \nabla\left(\partial_{3}^{3} \psi\right)^{2}\right. \\
& \left.+v \cdot \nabla \partial_{3} \psi\left(\partial_{3}^{3} \psi\right)^{2}+\partial_{3} v \cdot \nabla \psi\left(\partial_{3}^{3} \psi\right)^{2}\right\} d x d t \mid \\
\leq & C B_{T}^{3}\left(1+B_{T}^{2}\right) .
\end{aligned}
$$

In the last step above, we have also applied the following estimate,

$$
\left|\int_{0}^{T} \int\left\{2 \partial_{3} \psi \partial_{3}^{3} \psi\left[\partial_{3}^{3}(v \cdot \nabla \psi)-v \cdot \nabla \partial_{3}^{3} \psi\right]+\partial_{3} v \cdot \nabla \psi\left(\partial_{3}^{3} \psi\right)^{2}\right\} d x d t\right|
$$




$$
\begin{aligned}
\leq & \left|\int_{0}^{T} \int 2 \partial_{3} \psi \partial_{3}^{3} \psi\left(\partial_{3}^{3} v \cdot \nabla \psi+3 \partial_{3}^{2} v \cdot \nabla \partial_{3} \psi\right) d x d t\right|+\left|\int_{0}^{T} \int 6 \partial_{3} \psi \partial_{3}^{3} \psi \partial_{3} v_{h} \cdot \nabla_{h} \partial_{3}^{2} \psi d x d t\right| \\
& +\left|\int_{0}^{T} \int 7 \partial_{3} \psi \partial_{3} v_{3}\left(\partial_{3}^{3} \psi\right)^{2} d x d t\right|+\left|\int_{0}^{T} \int \partial_{3} v_{h} \cdot \nabla_{h} \psi\left(\partial_{3}^{3} \psi\right)^{2} d x d t\right| \\
\leq & C\|\nabla \psi\|_{L_{T}^{4}\left(L^{\infty}\left(\mathbb{R}^{3}\right)\right)}^{2}\left\|\partial_{3}^{3} \psi\right\|_{L_{T}^{\infty}\left(L^{2}\left(\mathbb{R}^{3}\right)\right)}\left\|\nabla^{3} v\right\|_{L_{T}^{2}\left(L^{2}\left(\mathbb{R}^{3}\right)\right)} \\
& +C\|\nabla \psi\|_{L_{T}^{4}\left(L^{\infty}\left(\mathbb{R}^{3}\right)\right)}\left\|\partial_{3}^{3} \psi\right\|_{L_{T}^{\infty}\left(L^{2}\left(\mathbb{R}^{3}\right)\right)}\left\|\nabla^{2} v\right\|_{L_{T}^{2}\left(L^{4}\left(\mathbb{R}^{3}\right)\right)}\left\|\nabla^{2} \psi\right\|_{L_{T}^{4}\left(L^{4}\left(\mathbb{R}^{3}\right)\right)} \\
& +C\left\|\partial_{3} \psi\right\|_{L_{T}^{\infty}\left(L^{\infty}\left(\mathbb{R}^{3}\right)\right)}\left\|\partial_{3}^{3} \psi\right\|_{L_{T}^{\infty}\left(L^{2}\left(\mathbb{R}^{3}\right)\right)}\left\|\partial_{3} v\right\|_{L_{T}^{2}\left(L^{\infty}\left(\mathbb{R}^{3}\right)\right)}\left\|\nabla_{h} \partial_{3}^{2} \psi\right\|_{L_{T}^{2}\left(L^{2}\left(\mathbb{R}^{3}\right)\right)} \\
& +C B_{T}^{4}\left(1+B_{T}\right)+C\left\|\partial_{3} v\right\|_{L_{T}^{2}\left(L^{\infty}\left(\mathbb{R}^{3}\right)\right)}\left\|\nabla_{h} \psi\right\|_{L_{T}^{2}\left(L^{\infty}\left(\mathbb{R}^{3}\right)\right)}\left\|\partial_{3}^{3} \psi\right\|_{L_{T}^{\infty}\left(L^{2}\left(\mathbb{R}^{3}\right)\right)}^{2} \\
\leq & C B_{T}^{4}\left(1+B_{T}\right) .
\end{aligned}
$$

The remaining parts have already shown to have the desired estimates. Thus we complete the proof of Lemma.

\section{Acknowledgements}

The research of F.H.Lin is partial supported by the NSF grants, DMS 1065964 and DMS 1159313. The research of T. Zhang is partially supported by NSF of China under Grants 11271322, 11331005 and 11271017, National Program for Special Support of Top-Notch Young Professionals, Program for New Century Excellent Talents in University NCET-11-0462, the Fundamental Research Funds for the Central Universities (2012QNA3001). Part of the work was done while the second author was visiting the Courant Institute Mathematical Sciences. T.Z. wants to thank the Courant Institute for the warm hospitality.

\section{References}

[1] C. Bardos, C. Sulem, P.-L. Sulem, Longtime dynamics of a conductive fluid in the presence of a strong magnetic field. Trans. Amer. Math. Soc. 305 (1988), no. 1, 175-191

[2] C.S. Cao, J.H. Wu, Global regularity for the 2D MHD equations with mixed partial dissipation and magnetic diffusion. Adv. Math. 226 (2011), no. 2, 1803-1822.

[3] P. Constantin, Complex fluids and Lagrangian particles. Topics in mathematical fluid mechanics, 1-21, Lecture Notes in Math., 2073, Springer, Heidelberg, 2013.

[4] P. Constantin, Remarks on complex fluid models. Mathematical aspects of fluid mechanics. 70-87, London Math. Soc. Lecture Note Ser., 402, Cambridge Univ. Press, Cambridge, 2012

[5] M. Doi and S. F. Edwards, The Theory of Polymer Dynamics. Oxford Science Publication, 1986.

[6] G. Duvaut, J.-L. Lions, Inéquations en thermoélasticité et magnétohydrodynamique, Arch. Rational Mech. Anal. 46 (1972) 241-279.

[7] U. Frisch, A. Pouquet, P. L. Sulem, and M. Meneguzzi, The dynamics of two-dimensional ideal MHD, J. Méc. Théor. Appl. 2 (1983), Special Suppl. 191-216.

[8] C. Guillopé and J. C. Saut, Existence results for the flow of viscoelastic fluids with a differential constitutive law, Nonlinear Anal., 15(1990), no. 9, 849-869.

[9] R. H. Kraichnan, Lagrangian-history closure approximation for turbulence. Phys. Fluids 8 (1965) $575-598$.

[10] P. R. Kramer, C. S. Peskin, and P. J. Atzberger, On the foundations of the stochastic immersed boundary method. Comput. Methods Appl. Mech. Engrg., 197(25-28): 2232-2249, 2008. 
[11] R. G. Larson. The Structure and Rheology of Complex Fluids. Oxford University Press, New York, 1999.

[12] F.H. Lin, Some analytical issues for elastic complex fluids, Comm. Pure Appl. Math. 65 (2012), no. 7, 893-919.

[13] F.H. Lin, L. Xu and P. Zhang, Global small solutions to 2-D incompressible MHD system, arXiv: 1302.5877 .

[14] F.H. Lin and P. Zhang, Global small solutions to an MHD-type system: the three-dimensional case, Comm. Pure Appl. Math., 67(2014), no. 4, 531-580.

[15] A. Majda, Compressible fluid flow and systems of conservation laws in several space variables. Springer-Verlag, New York, 1984.

[16] L. Nirenberg, An extended interpolation inequality, Ann. Scuola Norm. Sup. Pisa (3) 20 (1966) 733-737.

[17] M. Renardy, W. J. Hrusa, and J. A. Nohel, Mathematical Problems in Viscoelasticity, volume 35 of Pitman Monographs and Surveys in Pure and Applied Mathematics. Longman Scientific \& Technical, Harlow, 1987

[18] M. Sermange, R. Temam, Some mathematical questions related to the MHD equations, Comm. Pure Appl. Math. 36(1983), no. 5, 635-664 